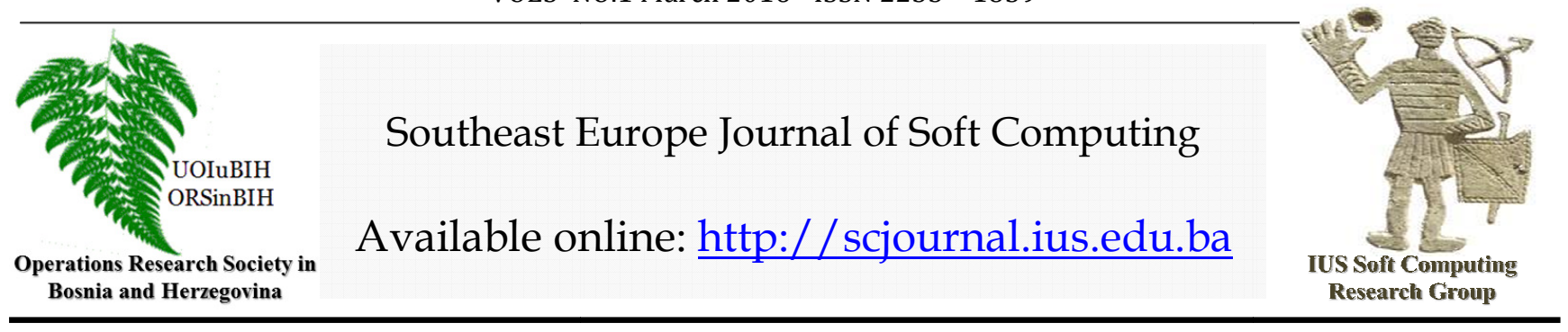

\title{
Fuzzy to Random Uncertainty Alignment
}

\author{
Migdat I. Hodžić \\ Santa Clara University, California, USA \\ International University of Sarajevo, Bosnia and Herzegovina \\ migdathodzic@gmail.com
}

Article Info

Article history:

Article received on 1 Mar. 2016

Received in revised form 17 Mar. 2016

Keywords:

Fuzzy distributions, Cumulative random distributions, Probability of random events, Fuzzy to

random uncertainty alignment,

Unimodal and multimodal fuzzy

data, Consistency principle

\begin{abstract}
The objective of this paper is to present new and simple mathematical approach to deal with uncertainty alignment between fuzzy and random data. In particular we present a method to describe fuzzy (possibilistic) distribution in terms of a pair (or more) of related random (probabilistic) events, both fixed and variable. Our approach uses basic properties of both fuzzy and random distributions. We show that the data fuzziness can be viewed as a non uniqueness of related random events. We also show how fuzzy-random consistancy principle can be given precise mathemtaical meaning. Various types of fuzzy distributions are examined, special cases considered, and several numerical examples presented.
\end{abstract}

\section{INTRODUCTION}

In studies of uncertain phenomena, several methods are employed. The two most widely used are random and fuzzy data approaches. They are typically described in terms of random and fuzzy distributions [4]. These two methods look at the uncertainty from different points of view. In literature one can find various terms for fuzzy data, such as possibilistic, soft, subjective, and similar [5], as opposed to random called probabilistic, hard, objective and the like. Historically, probability is defined in the context of some physical measurement and mathematically in terms of probability axioms by Kolmogorov [1] where probability space, events and associated probabilities are defined. Related notion of random variables are defined in terms of mappings from probabilistic space of events to real line, carrying an underlying probabilities from the original event space. Indexing with some independent variable, such as time, one can define random processes as dynamic versions of random variables. On the other hand fuzzy, possibilistic approach relates to some intuitive uncertain notion (often of human nature) of an underlying uncertain event with some confidence (presumption) levels defined. In fuzzy data there is no reference, at least not directly, to any experiment or hard measurement. It is more representation of our confidence level in an uncertain phenomenon. If a need arises to combine fuzzy and random data (such as in soft/hard data fusion applications) each distribution is typically handled individually for a specific problem at hand, and no rigorous mathematical methodologies exist for any meaningful uncertainty alignment between the two types of data. In a fundamental paper by Zadeh [2], a concept of possibilistic fuzzy distributions was introduced as opposed to random and probabilistic distributions. The possibilistic distribution is shown to be equivalent numerically to fuzzy membership function typically used in fuzzy data description. In another classic reference [4], various algebraic operations on fuzzy data are elaborated in details, as well as the methods as how to combine fuzzy and random data in meaningful ways. One obvious and an add hoc method is to normalize random data distribution to unity and combine it with the fuzzy data. Mathematically correct in principle, this method can be 
considered as a sort of uncertainty alignment from random to fuzzy data. Unfortunately the method is not practical because of loss of information in the process [4]. Also, in our opinion this method does not have any strong conceptual ground. Another approach is to define hybrid data which retains both fuzzy and random properties of original data. Two possible versions of the hybrid approach are described in [4]. First, one defines random fuzzy data where fuzzy distribution argument is "randomized" according to a probabilistic distribution density. Second, one can consider fuzzy random data where the value of random distribution density is fuzzified according to fuzzy data distribution [4]. Other methods have been considered as well. The subject of our paper is to consider fuzzy to random uncertainty alignment using very basic properties of both fuzzy and random distributions and associated probabilities. In our approach we use two step approach. First, we represent (decompose) fuzzy distributions via cumulative probabilistic distributions (CDF) instead of using probabilistic distribution densities (CDF derivative, which may not always exist). The immediate mathematical reason for that is the fact that cumulative random distributions are normalized to unity by the definition, just like fuzzy distributions. The other, conceptual reason, is that the possibilistic distribution resembles CDF more so than a distribution density. The second step uses basic probabilistic axioms whereas the CDFs are described in terms of some corresponding random event probabilities. For a unimodal fuzzy data the result is that the fuzzy distribution can be thought of as a combination of a fixed and a variable probability event. In the case of multimodal fuzzy data, this representation consists of a number of fixed and variable random events probabilities. We believe that our approach can bring about some interesting new avenues in treating fuzzy and random data, in particular in very important area of soft-hard (human-machine) data fusion. The paper is organized as follows. In Section 2 we briefly review basic probability results and related $\mathrm{CDF}$ definition and its properties. Section 3 summarizes important and related possibilistic (fuzzy) results including possibility theory axioms. In Section 4 we introduce our main fuzzy to random uncertainty alignment arguments using standard triangular fuzzy distribution (TFN). We describe two related probabilistic steps which result in a combination of fixed and variable probability (non unique randomness) description of a fuzzy distribution. In Section 5 we formalize and prove two key results, namely (i) probabilistic decomposition of an nmodal fuzzy distribution, and (ii) universal fuzzy to random uncertainty alignment law, which is also fuzzy presumption invariant. Section 6 presents additional fuzzy distribution examples to reinforce the applicability of our methodology. In Section 7 four numerical examples (symmetric TFN, non symmetric TFN, convex and non convex distributions) are presented showing fuzzy distributions in terms of fixed (unique) and variable (non unique) random events and related probabilities. The numerical results confirm our main results of Section 5. Conclusion and ideas for future work are given in Section 8. Finally, list of key references is given at the end of the paper.

\section{PROBABILITY, RANDOM VARIABLES AND CUMULATIVE DISTRIBUTIONS}

\subsection{Probability Theory}

In probabilistic (random experiments) we consider the occurrence of events (denoted by capital letters A, B) that are represented by sets. New events are obtained by combining old events, using proper set operations. Also, complex events can be expressed as combinations of a number of simple events. Sample space $S$ is assumed to be a set of all possible (countable) outcomes of the random experiment, with the empty set $\mathrm{O}$. Venn diagrams are used for working with sets, as well as random events [1] The events of interest (A, $A_{1}, A_{2}$, etc.) are the subsets of $S$, and they are assigned certain probabilities $P$. In this section we summarize basic probability axioms [1]:

$$
\begin{gathered}
0 \leq \mathrm{P}(\mathrm{A}) \leq 1, \mathrm{P}(\mathrm{S})=1, \mathrm{P}(\mathrm{O})=0 \\
\mathrm{P}\left(\mathrm{A}_{1} \mathrm{UA}_{2}\right)=\mathrm{P}\left(\mathrm{A}_{1}\right)+\mathrm{P}\left(\mathrm{A}_{2}\right)-\mathrm{P}\left(\mathrm{A}_{1} \cap \mathrm{A}_{2}\right)
\end{gathered}
$$

and conditional probability formulas:

$$
\mathrm{P}\left(\mathrm{A}_{1} \cap \mathrm{A}_{2}\right)=\mathrm{P}\left(\mathrm{A}_{1} / \mathrm{A}_{2}\right) \mathrm{P}\left(\mathrm{A}_{2}\right)=\mathrm{P}\left(\mathrm{A}_{2} / \mathrm{A}_{1}\right) \mathrm{P}\left(\mathrm{A}_{1}\right)
$$

When $A_{1} \cap A_{2}=0$, then per the above axioms $P\left(A_{1} \cap A_{2}\right)=0$, and we call two events $A_{1}$ and $A_{2}$ mutually exclusive. If $\mathrm{A}_{1} \mathrm{UA}_{2}=\mathrm{S}$ and two events are mutually exclusive, then $\mathrm{P}\left(\mathrm{A}_{1} \mathrm{UA}_{2}\right)=\mathrm{P}\left(\mathrm{A}_{1}\right)+\mathrm{P}\left(\mathrm{A}_{2}\right)=1$, and we call $\mathrm{A}_{1}$ and $\mathrm{A}_{2}$ complementary events, with $\mathrm{P}\left(\mathrm{A}_{1}\right)=1-\mathrm{P}\left(\mathrm{A}_{2}\right)=\mathrm{P}\left(\mathrm{A}_{2}{ }^{*}\right)$ and the symbol * indicates complementary event. For any event $\mathrm{A}$, the following holds:

$$
\mathrm{P}(\mathrm{A})+\mathrm{P}\left(\mathrm{A}^{*}\right)=1
$$

If the events are independent, then we have:

$$
\begin{gathered}
\mathrm{P}\left(\mathrm{A}_{1} \cap \mathrm{A}_{2}\right)=\mathrm{P}\left(\mathrm{A}_{1}\right) \mathrm{P}\left(\mathrm{A}_{2}\right) \\
\mathrm{P}\left(\mathrm{A}_{1} / \mathrm{A}_{2}\right)=\mathrm{P}\left(\mathrm{A}_{1}\right), \mathrm{P}\left(\mathrm{A}_{2} / \mathrm{A}_{1}\right)=\mathrm{P}\left(\mathrm{A}_{2}\right)
\end{gathered}
$$

Mutual exclusivity and independency do not imply each other. They would coincide in a trivial case when one of the probabilities $\mathrm{P}\left(\mathrm{A}_{1}\right)$ or $\mathrm{P}\left(\mathrm{A}_{2}\right)$ is zero.

\subsection{Random Variables}

A random variable $X(\xi)$ is a function that assigns a real number, to each outcome $\xi$ in the sample space $S$ of a random experiment [1]. Let us assume that a subset A (an event) is given in the sample space $S$ such that:

$$
\mathrm{A}=\{\xi: \mathrm{X}(\xi)=\mathrm{B}\}
$$

where B is a subset (an event) of real line R. A and B are equivalent events with the same probability:

$$
\mathrm{P}(\mathrm{X} \epsilon \mathrm{B})=\mathrm{P}(\mathrm{A})=\mathrm{P}(\{\xi: \mathrm{X}(\xi)=\mathrm{B}\})
$$

Often a random experiment outcome is already the numerical value $\xi$ we are interested in. Then we have $\mathrm{X}(\xi)$ $=\xi$. In general, a random variable $X$ is a function from $S$ to $R$ with the property that the set $A_{x}=\{\zeta: X(\zeta) \leq x\}$ is in the set of events of interest in $S$, for every $x$. Hence we require every set $A_{x}$ to have probability assigned to it. 


\subsection{Cumulative Random Distribution}

Consider a random experiment with sample space $\mathrm{S}$ and the subsets $A_{i}$ called events of interest. Then related notion of the cumulative distribution function (CDF) of $\mathrm{X}$ is defined as:

$$
\mathrm{F}_{\mathrm{X}}(\mathrm{x})=\mathrm{F}(\mathrm{x})=\mathrm{P}(\mathrm{X} \leq \mathrm{x}),-\infty \leq \mathrm{x} \leq+\infty
$$

which is a probability that $\mathrm{X}$ has a value in the set $(-\infty, \mathrm{x}]$, and hence it is a function of $x$. Note that (7) applies to both discrete and continuous random variables. We also recall that the distribution density, if exists, is defined as $\mathrm{f}(\mathrm{x})=$ $\mathrm{dF}(\mathrm{x}) / \mathrm{dx}$, and its integral is equal to 1 . Figure 1 shows uniform distribution example.

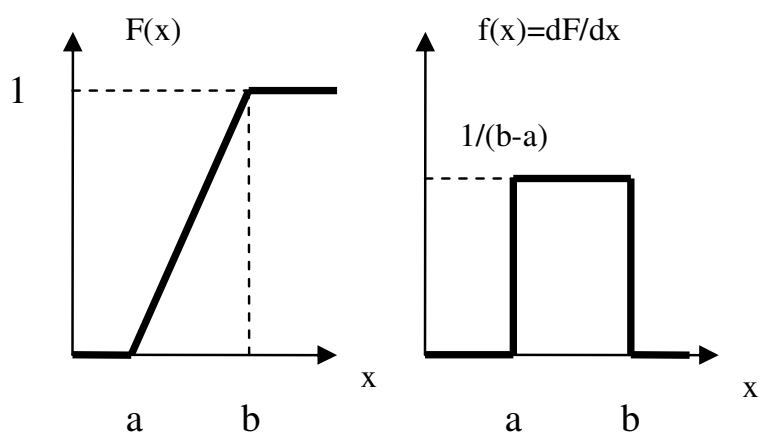

Figure 1. Uniform distribution

The properties of $\mathrm{F}(\mathrm{x})$ are summarized as [1]:

(i) $\quad 0 \leq \mathrm{F}(\mathrm{x}) \leq 1$

(ii) $\quad \lim _{\mathrm{x} \rightarrow \infty} \mathrm{F}(\mathrm{x})=1$

(iii) $\quad \lim _{x \rightarrow-\infty} F(x)=0$

(iv) $\quad \mathrm{F}(\mathrm{x})$ is a non decreasing function of $\mathrm{x}$ i.e. for $a \leq b, F(a) \leq F(b)$

(v) $\quad \mathrm{F}(\mathrm{x})$ is continuous from the right i.e. for $\delta>0, F(b)=\lim _{\delta \rightarrow 0} F(b+\delta)=F\left(b^{+}\right)$

$$
\mathrm{P}(\mathrm{a}<\mathrm{X} \leq \mathrm{b})=\mathrm{F}(\mathrm{b})-\mathrm{F}(\mathrm{a})
$$$$
\mathrm{P}(\mathrm{a} \leq \mathrm{X} \leq \mathrm{b})=\mathrm{F}(\mathrm{b})-\mathrm{F}\left(\mathrm{a}^{-}\right)
$$$$
\mathrm{P}(\mathrm{X}=\mathrm{b})=\mathrm{F}(\mathrm{b})-\mathrm{F}\left(\mathrm{b}^{-}\right)
$$

$$
\mathrm{P}(\mathrm{X}>\mathrm{x})=1-\mathrm{F}(\mathrm{x})
$$

The properties (vi) through (viii) indicate probabilities of semi open interval, closed interval, and complementary event, respectively. As stated earlier, in this paper we deal with the cumulative rather than density functions, both for mathematical as well as conceptual reasons.

\section{POSSIBILITY, FUZZY VARIABLES AND FUZZY DISTRIBUTIONS}

\subsection{Possibility Theory}

Possibility theory was introduced and developed early on by Zadeh [2] as an extension of the theory of fuzzy sets, and in the context of information meaning, in particular in the context of semantic variables and human soft (fuzzy) data. Hence the possibility is associated with fuzziness, either due to lack of knowledge on which possibility is based, or related to the subset (event) for which possibility is asserted. Since its inception, possibility theory was developed further, either in the spirit of Zadeh, or in the axiomatic framework of Demster-Shafer's Theory [3]. Another avenue is to view possibility as an upper probability bound, which allows study of possibility theory using the tools of imprecise probabilities. For the purposes of our paper, we limit ourselves to Zadeh's approach, with the aim to connect the possibility theory with the probability theory via fuzzy and variable random distributions. For the completeness sake we also summarize possibility axioms in the following section. Conceptually our approach appears to be similar to imprecise probability. The key difference is that we supply precise both lower and upper bounds on the "fuzziness" as opposed to just upper limit in the case of imprecise probability [6]. In our approach we explain the fuzziness as a non unique randomness. This approach came as a result of data fusion need in many relevant applications, where both hard data (objective, probabilistic, random, machine generated) measurements are obtained as well as a number of soft (subjective, possibilistic, fuzzy, human generated). In particular, possibility distribution is defined by Zadeh [2] as a fuzzy restriction on the values that may be assigned to a variable. Similar to probability theory, possibility as an extension of fuzzy sets is also based on set theory considerations. Say that A is a fuzzy subset of a universe of discourse $S=\{\xi\}$ (similar to space of events in probability), then the statement such as a proposition " $\mathrm{X}$ is A", and $\mathrm{X}$ takes values in $\mathrm{S}$, induces a possibility distribution $\Pi_{X}(\xi)$ which equates the possibility of $X$ taking value $\xi$ in $S$ to the fuzzy membership function $\mu_{A}(\xi)$, i.e. the compatibility of $\xi$ with A. Hence, $\Pi_{X}(\xi)=$ $\mu_{A}(\xi)$. In doing so we define $X$ as a fuzzy variable associated with the possibility distribution $\Pi_{X}$, similar to random variable associated with the probability in the original event space $\mathrm{S}$. For the simplicity of the notation we will use $\Pi(\mathrm{x})$ as the notation in this paper, with small $\mathrm{x}$ representing fuzzy variable $\mathrm{X}$ specific choice.

\subsection{Consistency Principle}

In the original Zadeh paper [2] there was no clarification as to the nature of the possibilistic vs. probabilistic distribution relationship, except for the "weak" connection between the two via his "consistency principle" defined as:

$$
\Gamma_{\mathrm{X}}=\sum_{\mathrm{i}=1, \ldots, \mathrm{n}} \mathrm{P}_{\mathrm{i}} \Pi_{\mathrm{i}}=\mathrm{P}_{1} \Pi_{1}+\mathrm{P}_{2} \Pi_{2}+\ldots+\mathrm{P}_{\mathrm{n}} \Pi_{\mathrm{n}}
$$

where the variable $\mathrm{X}$ can be interpreted both as probabilistic and possibilistic, with the corresponding distributions consisting of the same number of choices in the interval of interest. The idea behind the consistency principle comes from an intuitive observation that reducing the possibility of an event tends to reduce its probability. The opposite may not hold. The problem with this definition is that it is not clear what do we do with the value $\Gamma_{\mathrm{X}}$. The original Zadeh's idea for the consistency principle as given in (9) idea may be useful when possibility is known about $\mathrm{X}$ but not the probability. Our paper addresses that issue via uncertainty alignment idea. 
In Section 5 we describe an alternative definition of consistency principle which better reflects "consistency" idea between random and fuzzy variable distributions. This definition also lends itself to a precise mathematical and quantitative treatment, and it can be of great use in decision making process where data is inherently soft and subjective. One particular area of interest is humanmachine (soft-hard) data fusion. In Section 5 we prove that the consistency principle and "weak" connection can be clarified in a very precise mathematical way.

\subsection{Axiomatic Possibility Theory}

Following original work by Zadeh other researchers developed possibility theory idea further [3], [7], [8]. The following is a list of basic possibility axioms which are based on the original Zadeh work:

$$
0 \leq \Pi(\mathrm{A}) \leq 1, \quad \Pi(\mathrm{S})=1, \Pi(\mathrm{O})=0
$$

where $\mathrm{O}$ is an empty set and $\mathrm{S}$ is a universe of discourse, a finite or countably infinite set, with all subsets to which we can assign possibility measure $\Pi(x)$. Note that in the context of Zadeh notion of possibility, these are all fuzzy sets which induce fuzzy restriction upon the values of the fuzzy variable. For any two fuzzy subsets $A_{1}$ and $A_{2}$ of $S$, we have:

$$
\begin{aligned}
& \Pi\left(\mathrm{A}_{1} \mathrm{UA}_{2}\right)=\max \left[\Pi\left(\mathrm{A}_{1}\right), \Pi\left(\mathrm{A}_{2}\right)\right] \\
& \Pi\left(\mathrm{A}_{1} \cap \mathrm{A}_{2}\right) \leq \min \left[\Pi\left(\mathrm{A}_{1}\right), \Pi\left(\mathrm{A}_{2}\right)\right]
\end{aligned}
$$

We note that the operations (11) and (12) are simpler than the corresponding probability operations in (2) and (3). In addition to possibility, there is a corresponding notion of necessity N(A) of a (fuzzy) event A [3]. For any A, $A_{1}$ and $\mathrm{A}_{2}$ we have:

$$
\begin{gathered}
\mathrm{N}(\mathrm{A})=1-\Pi\left(\mathrm{A}^{*}\right), \quad \mathrm{N}(\mathrm{A}) \leq \Pi(\mathrm{A}) \\
\mathrm{N}\left(\mathrm{A}_{1} \cap \mathrm{A}_{2}\right) \leq \min \left[\mathrm{N}\left(\mathrm{A}_{1}\right), \mathrm{N}\left(\mathrm{A}_{2}\right)\right] \\
\operatorname{Max}\left[\Pi\left(\mathrm{A}_{1}\right), \Pi\left(\mathrm{A}_{2}\right)\right]=1 \\
\operatorname{Min}\left[\mathrm{N}\left(\mathrm{A}_{1}\right), \mathrm{N}\left(\mathrm{A}_{2}\right)\right]=0 \\
\mathrm{~N}(\mathrm{~A})+\mathrm{N}\left(\mathrm{A}^{*}\right) \leq 1 \\
\Pi(\mathrm{A})+\Pi\left(\mathrm{A}^{*}\right) \geq 1
\end{gathered}
$$

In a follow up work we will investigate how the two sets of axioms, probability and possibility, are related in a view of fuzzy to random (possibilistic to probabilistic), uncertainty alignment approach of this paper.

\section{POSSIBILISTIC TO PROBABILISTIC UNCERTAINTY ALIGNMENT}

We proceed by considering a typical triangular fuzzy distribution number (TFN) given in Figure 2 with the interval of interest $[a, b, c]$ for any $a, b$ and $c$, and the corresponding possibilistic distribution $\Pi(\mathrm{x})$ numerically equivalent to the fuzzy membership function $\mu$ (x) [2]. At this point we will not write equations of the segments of the distribution $\Pi(\mathrm{x})$. This will be done in Section 7 with numerical examples. We want simply to illustrate the approach in the simplest possible way, for conceptual reasons. Note that our approach can be applied to any other fuzzy variable, unimodal or multimodal, symmetric or not, normal or non normal, convex or non convex, trapezoidal or arbitrary shaped fuzzy distribution. The key is that the cumulative function properties given in (8) are satisfied. Section 6 shows additional examples of fuzzy distributions. Next step is to define a pair of CDFs such as in Figure 1 to "decompose" П(x):

$$
\Pi(\mathrm{x})=\mathrm{F}_{1}(\mathrm{x})-\mathrm{F}_{2}(\mathrm{x})
$$

where $F_{1}$ and $F_{2}$ are shown in Figure 3 bellow. They both satisfy properties given in (8), and both indicate uniform probabilistic distributions. Note that if the TFN is not normalized in Figure 2, that would not pose any problem for the decomposition. Namely $F_{1}$ and $F_{2}$ would have corresponding jumps extending to 1 , per properties (v) (vii) given in (8), to satisfy the conditions of continuity

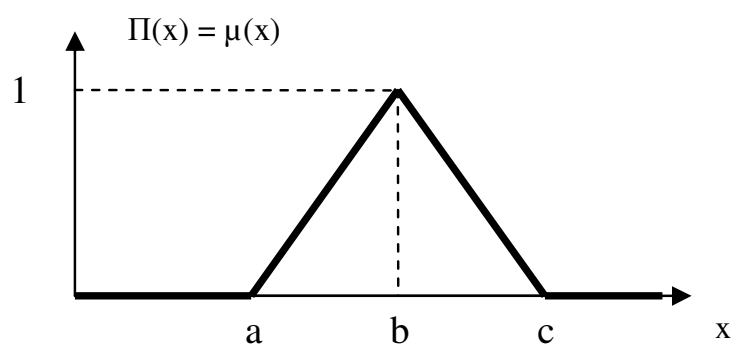

Figure 2. Triangular fuzzy number (TFN)
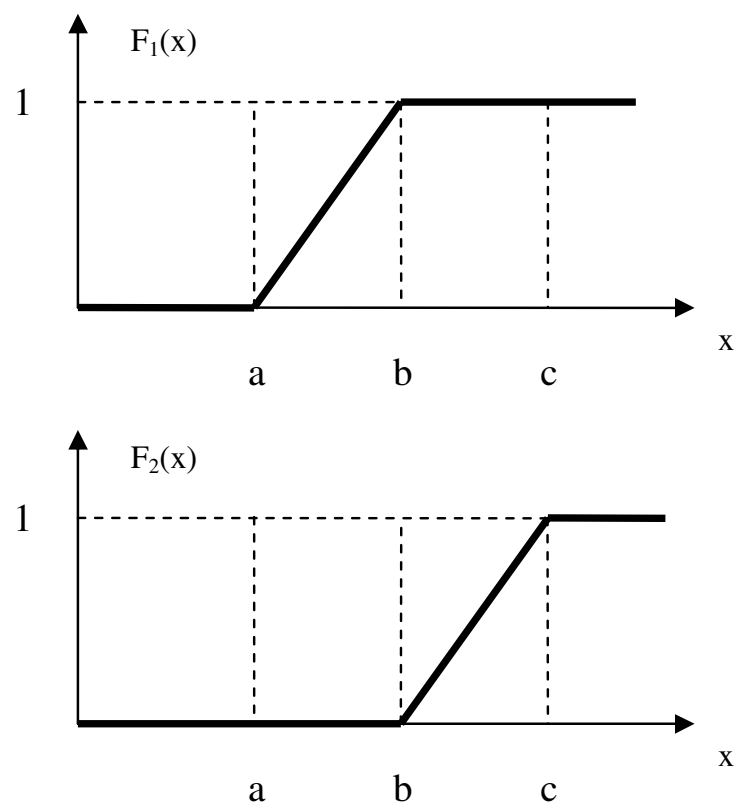

Figures 3. Cumulative functions $F_{1}$ and $F_{2}$

and their maximum at 1 . At this point we have to take another "probabilistic" step to refine the decomposition (14). We note that the cumulative function $\mathrm{F}$ is defined in terms of a random event probability given in (8). Next we recall (2) in Section 2.1 which relates probabilities of some events $A_{1}$ and $A_{2}$, the subsets of a certain event $S$. 


\section{Hodzic, M./ Southeast Europe Journal of Soft Computing Vol.5 No.1 March 2016 (58-67)}

Let us rewrite (2) as follows:

$$
P\left(A_{1}\right)-P\left(A_{1} \cap A_{2}\right)=P\left(A_{1} U A_{2}\right)-P\left(A_{2}\right)
$$

Each side of the Equation (15) satisfies basic probabilistic axioms in (1). The nice property of (15) is that both sides have negative terms, as does Equation (14). We proceed with two cases:

Case 1. First we equate left side of (15) with (14):

$$
\Pi(x)=F_{1}(x)-F_{2}(x)=P\left(A_{1}\right)-P\left(A_{1} \cap A_{2}\right)
$$

where the event $A_{1}$ uniquely corresponds to the form given in (7), i.e. $A_{1}=\{X \leq x\}=\{\zeta: X(\zeta) \leq x\}$ for a random experiment $\zeta$. The event $\mathrm{A}_{1} \cap \mathrm{A}_{2}$ and $\mathrm{A}_{2}$ are to be determined. The two cumulative distributions are uniquely defined as:

$$
F_{1}(x)=P\left(A_{1}\right), F_{2}(x)=P\left(A_{1} \cap A_{2}\right)
$$

(Figure 3). What is not uniquely determined is the event $A_{2}$ because different $\mathrm{A}_{2}$ can produce the same sets intersection $A_{1} \cap A_{2}$. Note that we assume that the random variable $X$ can be interpreted both as a probabilistic (random) as well as possibilistic (fuzzy) variable. In Zadeh paper [2], the same assumption was made in terms of his "consistency" principle. The assumption makes sense for we are looking to analyze and uncertainty align related possibilistic and probabilistic distributions, or "consistent" ones. To further clarify the event $\mathrm{A}_{2}$, a little reflection on the set theory (Venn diagram, Figure 4), the fact that $A_{1}$ and $A_{2}$ are the subsets of $S$, plus our interest in the intersection $A_{1} \cap A_{2}$, brings us to the following condition on $\mathrm{P}\left(\mathrm{A}_{2}\right)$ :

$$
\mathrm{P}\left(\mathrm{A}_{1} \cap \mathrm{A}_{2}\right) \leq \mathrm{P}\left(\mathrm{A}_{2}\right) \leq 1-\Pi(\mathrm{x})
$$

producing the same intersection $\mathrm{P}\left(\mathrm{A}_{1} \cap \mathrm{A}_{2}\right)$, and the right hand side $1-\Pi(\mathrm{x})=\Pi^{*}(\mathrm{x})$ represents a complementary fuzzy distribution to $\Pi(\mathrm{x})$, which upholds the last condition in (13) with the equality sign, due to probabilistic interpretation in (14) and (3). Figures 5 summarize $\mathrm{P}\left(\mathrm{A}_{2}\right)$ bounds given in (18), using $\mathrm{P}\left(\mathrm{A}_{1} \cap \mathrm{A}_{2}\right)$

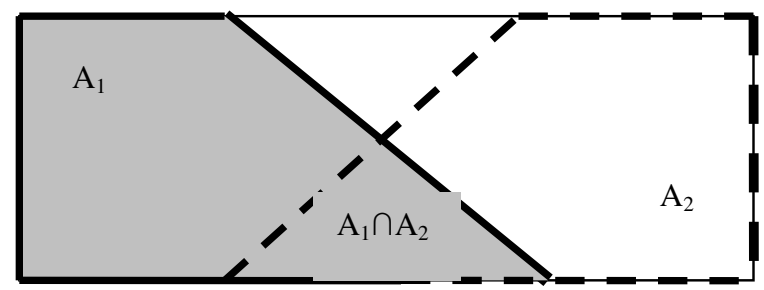

Figure 4. Venn diagram for two sets $A_{1}$ and $A_{2}$

in Figure 3. The gray area in $\mathrm{P}\left(\mathrm{A}_{2}\right)$ indicates non unique choices for the event $\mathrm{A}_{2}$ and the probability $\mathrm{P}\left(\mathrm{A}_{2}\right)$. Few possible choices are indicated by solid lines. They will all generate the same $F_{2}=P\left(A_{1} \cap A_{2}\right)$. Note that the grey (non unique $A_{2}$ ) area corresponds to $\mathrm{x}<\mathrm{b}$, while $\mathrm{A}_{2}$ is uniquely defined for $\mathrm{b} \leq \mathrm{x}$, due to a simultaneous action of conditions in (18). One can consider the interplay of unique $\mathrm{P}\left(\mathrm{A}_{1}\right)$ and non unique $\mathrm{P}\left(\mathrm{A}_{1} \cap \mathrm{A}_{2}\right)$, i.e. $\mathrm{P}\left(\mathrm{A}_{2}\right)$, equivalent to "fuzziness" of the left hand side of $\Pi(x)$, when $\mathrm{x}<\mathrm{b}$, Figure 2 . In the next subsection we consider Case 2 which will have the same effect on the right hand side of $\Pi(x)$.

Case 2. In this case we equate right side of (15) with (14) and write:

$$
\Pi(\mathrm{x})=\mathrm{F}_{1}(\mathrm{x})-\mathrm{F}_{2}(\mathrm{x})=\mathrm{P}\left(\mathrm{A}_{1} \mathrm{UA}_{2}\right)-\mathrm{P}\left(\mathrm{A}_{2}\right)
$$

where the event $A_{2}$ uniquely corresponds to the form given in (8), i.e. $A_{2}=\{X \leq x\}=\{\zeta: X(\zeta) \leq x\}$ for a random experiment $\zeta$. The event $\mathrm{A}_{1} \mathrm{UA}_{2}$ and $\mathrm{A}_{1}$ are to be determined. The two cumulative distributions are uniquely defined as:

$$
F_{1}(x)=P\left(A_{1} U_{2}\right), F_{2}(x)=P\left(A_{2}\right)
$$

Note that the forms of $F_{1}$ and $F_{2}$ are same as before (Figure $3)$, but we interpret them differently, i.e. as $\mathrm{P}\left(\mathrm{A}_{1} \mathrm{UA}_{2}\right)$ and $\mathrm{P}\left(\mathrm{A}_{2}\right)$ respectively. Now event $\mathrm{A}_{1}$ is not uniquely determined because different $A_{1}$ can produce the same sets unions $\mathrm{A}_{1} \mathrm{UA}_{2}$. As in the Case 1, we assume that the random variable $\mathrm{X}$ can be interpreted both as a random
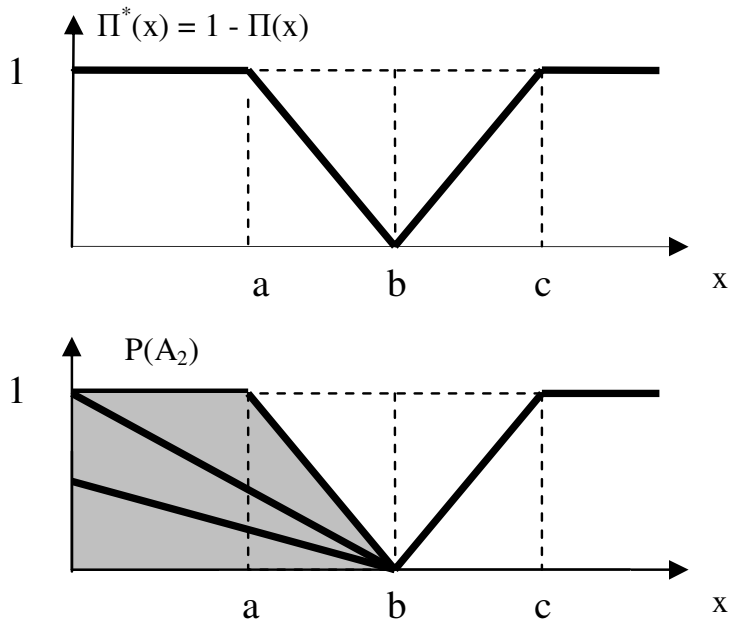

Figures 5. Case 1: Bounds on $\mathrm{P}\left(\mathrm{A}_{2}\right)$

(probabilistic) as well as fuzzy (possibilistic) variable. To further clarify $A_{1}$, we use Figure 4 , and consider union $\mathrm{A}_{1} \mathrm{UA}_{2}$, which produces the following condition on $\mathrm{P}\left(\mathrm{A}_{1}\right)$ :

$$
\Pi(\mathrm{x}) \leq \mathrm{P}\left(\mathrm{A}_{1}\right) \leq \mathrm{P}\left(\mathrm{A}_{1} \mathrm{UA}_{2}\right)
$$

Figures 6 summarize $P\left(A_{1}\right)$ bounds given in (21), and using $\mathrm{P}\left(\mathrm{A}_{1} \mathrm{UA}_{2}\right)$ in Figure 3 . The gray area in $\mathrm{P}\left(\mathrm{A}_{1}\right)$ 
indicates its non unique choices. Possible choices are indicated by solid lines. They will all generate the same $\mathrm{F}_{1}=\mathrm{P}\left(\mathrm{A}_{1} \mathrm{UA}_{2}\right)$. Note that the grey area corresponds to $\mathrm{b} \leq$ $\mathrm{x}$, while $\mathrm{P}\left(\mathrm{A}_{1}\right)$ is uniquely defined for $\mathrm{x}<\mathrm{b}$, due to $\mathrm{a}$ simultaneous action of conditions (21). As in the Case 1, one can consider that the interplay of unique $\mathrm{A}_{2}$ and $\mathrm{P}\left(\mathrm{A}_{2}\right)$ and non unique $\mathrm{A}_{1}$ and $\mathrm{P}\left(\mathrm{A}_{1} \mathrm{UA}_{2}\right)$ is equivalent to "fuzziness" of the right hand side of $\Pi(x)$, when $b \leq x$, Figure 2. By combining Case 1 and Case 2, we conclude that non unique choices for $\mathrm{A}_{1}$ and $\mathrm{A}_{2}$ and their corresponding probabilities $\mathrm{P}\left(\mathrm{A}_{1}\right)$ and $\mathrm{P}\left(\mathrm{A}_{2}\right)$, correspond to the non zero part of the distribution $\Pi(\mathrm{x})$. Outside of that, when $\Pi(x)=0$, they can be considered independent or exclusive for the trivial (limiting) cases of probabilities
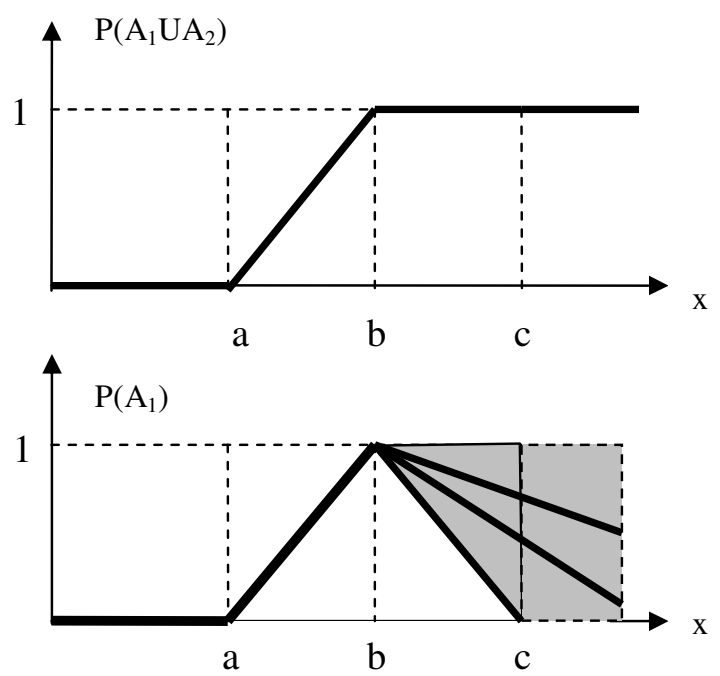

Figures 6. Case 2: Bounds on $\mathrm{P}\left(\mathrm{A}_{1}\right)$

0 or 1 . In a conclusion, the fuzzy distribution $\Pi(x)$ is generated by an action of random events $A_{1}$ and $A_{2}$, with respective probabilities $\mathrm{P}\left(\mathrm{A}_{1}\right)$ and $\mathrm{P}\left(\mathrm{A}_{2}\right)$, which switch between unique and non unique depending of the fuzzy argument $\mathrm{x}$, per Table 1 , where we used the notation $\mathrm{P}_{1}=\mathrm{P}\left(\mathrm{A}_{1}\right)$ and $\mathrm{P}_{2}=\mathrm{P}\left(\mathrm{A}_{2}\right)$. Note that the non zero $\Pi(\mathrm{x})$ corresponds to the gray shaded areas in Table 1.

Table 1. $\Pi(x)$ in terms of random events $A_{1}$ and $A_{2}$

\begin{tabular}{|c|c|c|c|}
\hline $\mathrm{x}$ & $\mathrm{A}_{1}$ & $\mathrm{~A}_{2}$ & $\mathrm{~A}_{1}$ vs. $\mathrm{A}_{2}$ \\
\hline $0 \leq \mathrm{x}<\mathrm{a}$ & Unique & Non & Exclusive \\
& $\mathrm{P}_{1}=0$ & $\begin{array}{c}\text { unique } \\
\left(\mathrm{P}_{1}=0, \mathrm{P}_{2}=1\right)\end{array}$ \\
& & $0 \leq \mathrm{P}_{2}$ & $\begin{array}{c}\text { Independent } \\
\leq 1\end{array}$ \\
& & $\left.\mathrm{P}_{1}=\mathrm{P}_{2}=0\right)$ \\
\hline $\mathrm{a} \leq \mathrm{x}<\mathrm{b}$ & Unique & $\begin{array}{c}\text { Non } \\
\text { unique }\end{array}$ & $\mathrm{P}_{1}+\mathrm{P}_{2} \leq 1$ \\
& & & \\
\hline
\end{tabular}

\begin{tabular}{|c|c|c|c|}
\hline $\mathrm{b} \leq \mathrm{x}<\mathrm{c}$ & $\begin{array}{c}\text { Non } \\
\text { unique }\end{array}$ & Unique & $\begin{array}{c}\Pi=1-\mathrm{P}_{2} \\
\mathrm{P}_{1}+\mathrm{P}_{2} \leq 1\end{array}$ \\
\hline $\mathrm{c} \leq \mathrm{x}$ & Non & Unique & Exclusive \\
& unique & $\mathrm{P}_{2}=1$ & $\left(\mathrm{P}_{1}=0, \mathrm{P}_{2}=1\right)$ \\
& $0 \leq \mathrm{P}_{1} \leq 1$ & & Independent \\
& & & $\left(\mathrm{P}_{1}=\mathrm{P}_{2}=1\right)$ \\
& & & \\
\hline
\end{tabular}

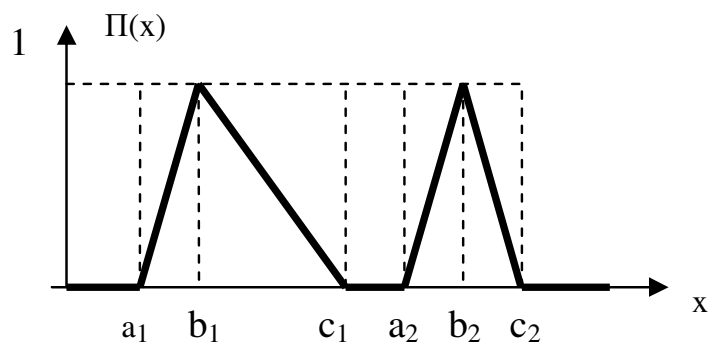

Figure 7. Bi modal TFN

\section{MAIN RESULTS}

In this section we summarize Section 4 results in the form of two Theorems and two Corollaries. Various types of fuzzy distributions, other than TFNs, can be handled by our approach. These distributions can be non convex, non normalized, and of various other shapes, symmetric and non symmetric, both unimodal and multimodal. Figure 7 shows a bi modal fuzzy distribution consisting of a pair of TFNs. In this case the decomposition of $\Pi(x)$ consists of a two pairs of cumulative probabilistic distributions:

$$
\Pi(\mathrm{x})=\mathrm{F}_{1}(\mathrm{x})-\mathrm{F}_{2}(\mathrm{x})+\mathrm{F}_{3}(\mathrm{x})-\mathrm{F}_{4}(\mathrm{x})
$$

or in terms of probabilities, extending (16), (17), (19) and (20) to bi modal case:

$$
\begin{gathered}
\Pi(x)=P\left(A_{1}\right)-P\left(A_{1} \cap A_{2}\right)+P\left(A_{3}\right)-P\left(A_{3} \cap A_{4}\right) \\
=P\left(A_{1} U_{2}\right)-P\left(A_{2}\right)+P\left(A_{3} U_{4}\right)-P\left(A_{4}\right)
\end{gathered}
$$

By an induction extension of (22), for a " $n$ " modal TFN we have the following general result:

Theorem 1. Fuzzy n-modal distribution function $\Pi(x)$ can be decomposed as a difference of sums of probabilistic cumulative distributions:

$$
\Pi(\mathrm{x})=\sum \mathrm{F}_{\mathrm{i}}(\mathrm{x})-\sum \mathrm{F}_{\mathrm{j}}(\mathrm{x})
$$

with $\mathrm{i}=2 \mathrm{k}-1, \mathrm{j}=2 \mathrm{k}, \mathrm{k}=1,2, \ldots, \mathrm{n}$, where the odd functions amount for rising portion of fuzzy distribution and even for the falling portion. In terms of probabilities, by the extension of (24) we have the following:

Corollary 1. Fuzzy n-modal distribution function given in Theorem 1 can be further expressed as a difference of sums of probabilities: 


$$
\begin{aligned}
\Pi(\mathrm{x}) & =\sum \mathrm{P}\left(\mathrm{A}_{\mathrm{i}}\right)-\sum \mathrm{P}\left(\mathrm{A}_{\mathrm{i}} \cap \mathrm{A}_{\mathrm{j}}\right) \\
& =\sum \mathrm{P}\left(\mathrm{A}_{\mathrm{i}} \mathrm{UA}_{\mathrm{j}}\right)-\sum \mathrm{P}\left(\mathrm{A}_{\mathrm{j}}\right)
\end{aligned}
$$

with $\mathrm{i}=2 \mathrm{k}-1$ and $\mathrm{j}=2 \mathrm{k}, \mathrm{k}=1,2, \ldots, \mathrm{n}$. Using (24) and (25), as well as (18) and (21), we now state the following key result which relates a fuzzy distribution and a set of changes in variable exclusive event probabilities.

Theorem 2. Any multimodal fuzzy distribution $\Pi(\mathrm{x})$ can be expressed in terms of the following fuzzy presumptioninvariant and $\mathrm{x}$-invariant universal fuzzy-random uncertainty alignment law:

$$
\Pi(\mathrm{x})+\sum \Delta \mathrm{P}\left(\mathrm{A}_{\mathrm{i}}\right)+\sum \Delta \mathrm{P}\left(\mathrm{A}_{\mathrm{j}}\right)=1, \text { for any } \mathrm{x}
$$

with $\mathrm{i}=2 \mathrm{k}-1, \mathrm{j}=2 \mathrm{k}, \mathrm{k}=1,2, \ldots, \mathrm{n}$, where the range in probability is $\Delta \mathrm{P}\left(\mathrm{A}_{\mathrm{k}}\right)=\mathrm{P}\left(\mathrm{A}_{\mathrm{k}}\right)_{\mathrm{M}}-\mathrm{P}\left(\mathrm{A}_{\mathrm{k}}\right)_{\mathrm{m}}$ per (18), "M" is for maximum, " $\mathrm{m}$ " is for minimum value. For simplicity, we did not burden the notation with stating dependency of $\Delta \mathrm{P}$ 's on $\mathrm{x}$. The key feature of Theorem 2 is that it holds for any $\mathrm{x}$ and any presumption level of $\Pi(\mathrm{x})$. We prove the case when $n=1$, for TFN in Figure 2. The proof for any $n$ and any $\Pi(x)$ is straightforward. From (17), (18) and (21) we obtain:

$$
\begin{aligned}
& \text { Case } 1(\mathrm{x}<\mathrm{b}) \mathrm{P}\left(\mathrm{A}_{1}\right)_{\mathrm{m}}=\mathrm{P}\left(\mathrm{A}_{1}\right)_{\mathrm{M}} \\
& \Delta \mathrm{P}\left(\mathrm{A}_{1}\right)=\mathrm{P}\left(\mathrm{A}_{1}\right)_{\mathrm{M}}-\mathrm{P}\left(\mathrm{A}_{1}\right)_{\mathrm{m}}=0 \\
& \mathrm{P}\left(\mathrm{A}_{2}\right)_{\mathrm{M}}=1-\Pi(\mathrm{x}) \\
& \mathrm{P}\left(\mathrm{A}_{2}\right)_{\mathrm{m}}=0 \\
& \Delta \mathrm{P}\left(\mathrm{A}_{2}\right)=\mathrm{P}\left(\mathrm{A}_{2}\right)_{\mathrm{M}}-\mathrm{P}\left(\mathrm{A}_{2}\right)_{\mathrm{m}}=1-\Pi(\mathrm{x})
\end{aligned}
$$

Case $2(b \leq x)$ :

$$
\begin{aligned}
& \mathrm{P}\left(\mathrm{A}_{1}\right)_{\mathrm{m}}=\Pi(\mathrm{x}) \\
& \mathrm{P}\left(\mathrm{A}_{1}\right)_{\mathrm{M}}=1 \\
& \Delta \mathrm{P}\left(\mathrm{A}_{1}\right)=\mathrm{P}\left(\mathrm{A}_{1}\right)_{\mathrm{M}}- \mathrm{P}\left(\mathrm{A}_{1}\right)_{\mathrm{m}}=1-\Pi(\mathrm{x}) \\
& \mathrm{P}\left(\mathrm{A}_{2}\right)_{\mathrm{m}}=\mathrm{P}\left(\mathrm{A}_{2}\right)_{\mathrm{X}} \\
& \Delta \mathrm{P}\left(\mathrm{A}_{2}\right)=\mathrm{P}\left(\mathrm{A}_{2}\right)_{\mathrm{X}}-\mathrm{P}\left(\mathrm{A}_{2}\right)_{\mathrm{m}}=0
\end{aligned}
$$

Note that the point " $b$ " is a middle $(\max )$ point of a TFN, or any other unimodal fuzzy distribution. Replacing (28) and (29) into (26), for $n=1$, we obtain:

$$
\Pi(\mathrm{x})+\Delta \mathrm{P}\left(\mathrm{A}_{1}\right)+\Delta \mathrm{P}\left(\mathrm{A}_{2}\right)=1
$$

which holds across the full range of argument x. Another form of the Equation (29) is:

$$
\begin{aligned}
\Pi(\mathrm{x}) & =1-\left[\Delta \mathrm{P}\left(\mathrm{A}_{1}\right)+\Delta \mathrm{P}\left(\mathrm{A}_{2}\right)\right] \\
& =1-\Pi^{*}(\mathrm{x})
\end{aligned}
$$

where:

$$
\Pi^{*}(\mathrm{x})=\Delta \mathrm{P}\left(\mathrm{A}_{1}\right)+\Delta \mathrm{P}\left(\mathrm{A}_{2}\right)
$$

represents fuzzy complement to $\Pi(\mathrm{x})$, assuming equality in the last condition in (13). One can interpret this result as the "randomness" pool left to form fuzzy distribution to a random certainty. This also means that for higher "presumption" levels, near 1, corresponding randomness pool is smaller (less uncertainty to adjust) and for lower "presumption" levels it is larger (more uncertainty to adjust). Examples in Section 6 and Figures 9 show that clearly. We have the following result based on Theorem 2:
Corollary 2. Any fuzzy distribution derivative $\mathrm{d} \Pi(\mathrm{x}) / \mathrm{dx}$ can be expressed for any argument $\mathrm{x}$ as a universal fuzzyrandom uncertainty change law:

$$
\mathrm{d} \Pi(\mathrm{x}) / \mathrm{dx}+\sum \mathrm{d}\left[\Delta \mathrm{P}\left(\mathrm{A}_{\mathrm{i}}\right)\right] / \mathrm{dx}+\sum \mathrm{d}\left[\Delta \mathrm{P}\left(\mathrm{A}_{\mathrm{j}}\right)\right] / \mathrm{dx}=0
$$

and $\mathrm{i}=2 \mathrm{k}-1, \mathrm{j}=2 \mathrm{k}, \mathrm{k}=1,2, \ldots, \mathrm{n}$. For $\mathrm{n}=1$, we have

$$
\mathrm{d} \Pi(\mathrm{x}) / \mathrm{dx}+\mathrm{d}\left[\Delta \mathrm{P}\left(\mathrm{A}_{1}\right)\right] / \mathrm{dx}+\mathrm{d}\left[\Delta \mathrm{P}\left(\mathrm{A}_{2}\right)\right] / \mathrm{dx}=0
$$

Due to the fact that the changes in two probabilities $\Delta \mathrm{P}\left(\mathrm{A}_{1}\right)$ and $\Delta \mathrm{P}\left(\mathrm{A}_{2}\right)$ are not zero at different arguments $\mathrm{x}$ (Cases 1,2), (33) reduces to a very simple fact:

$$
\mathrm{d} \Pi(\mathrm{x}) / \mathrm{dx}=-\mathrm{d}\left[\Delta \mathrm{P}\left(\mathrm{A}_{\mathrm{i}}\right)\right] / \mathrm{dx}
$$

where $\Delta \mathrm{P}\left(\mathrm{A}_{\mathrm{i}}\right)$ corresponds to either $\Delta \mathrm{P}\left(\mathrm{A}_{1}\right)$ for $\mathrm{i}=1$, or to $\Delta \mathrm{P}\left(\mathrm{A}_{2}\right)$ for $\mathrm{i}=2$, depending on $\mathrm{x}$ value. Simply stated, (34) says that the change in fuzzy distribution is the opposite of random probability change. This is also clearly confirmed in Section 6 with four numerical examples, and Figures 9.

Consistency principle. Referring back to Zadeh "consistency" principle [2], as given in (9) for an unimodal fuzzy distribution, one can re interpret it in the light of our Theorem 2 which holds for any multimodal fuzzy distribution, any presumption level and any argument $\mathrm{x}$, and it has a clear (conceptual and numeric) message, not only intuitive rationale as in [2]. We can consider it as a "fuzzy-random uncertainty balance principle" and it can be used instead of "consistency principle" given in (9). Another view of the principle could be given in a slightly changed definition instead of (9), where instead of multiplying $\mathrm{P}_{\mathrm{i}}$ and $\Pi_{\mathrm{i}}$, we use the sum of $\Delta \mathrm{P}_{\mathrm{i}}$ and $\Pi_{\mathrm{i}}$ in the spirit of Theorem 2, where " $i$ " now points to a different $x$, that is $\mathrm{x}_{\mathrm{i}}$ :

$$
\Gamma_{\mathrm{X}}=\sum_{\mathrm{i}=1, \ldots, \mathrm{n}}\left(\Delta \mathrm{P}_{\mathrm{i}}+\Pi_{\mathrm{i}}\right)=\mathrm{n}
$$

Now the consistency principle has a definite numerical meaning as well. Note that $\Delta \mathrm{P}_{\mathrm{i}}$ is either $\Delta \mathrm{P}_{1}$ or $\Delta \mathrm{P}_{2}$ for they are never different than zero at the same time.

\section{OTHER FUZZY DISTRIBUTIONS}

Figures 8 show other types of fuzzy distributions which can be handled by our approach. The first one is a trapezoidal distribution which can be decomposed using a pair of CDFs. The second one is a bimodal and a combination of two distributions put together (gray area can belong to either). It can be decomposed by using two pairs of CDFs. The next one is a convex distribution with the maximum at "b". It can be decomposed by a pair of CDFs, with the break at "b". The last one is a concave example. First two fuzzy distributions consist of uniform random distributions, and the last two are not uniform. In Section 6 we show four numerical examples, two uniform, two non uniform. In every case the continuity conditions given in (8) must be observed when $\Pi(\mathrm{x})$ is expressed in terms of Theorem 1 . 

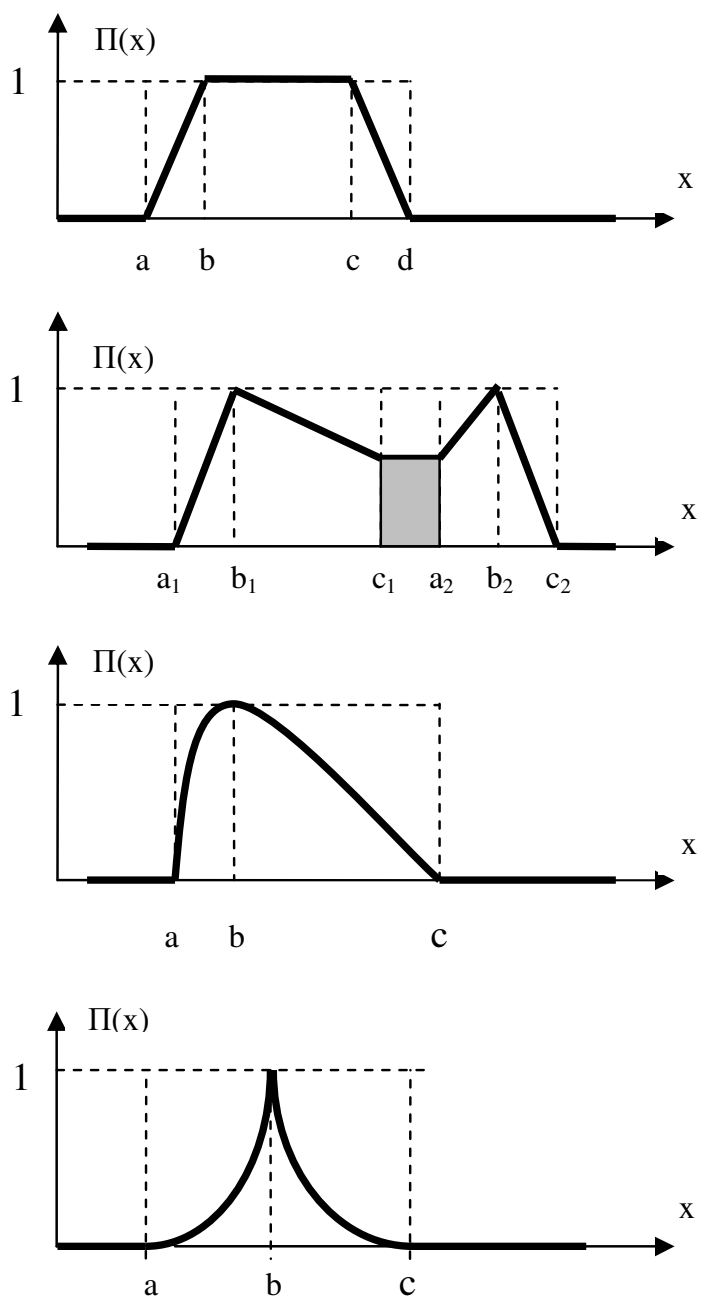

Figure 8. Other fuzzy distributions

\section{NUMERICAL EXAMPLES}

In this section we consider four numerical examples which illustrate the main results of the paper. Figure 2 and Equation (14) give a simple TFN decomposition with the CDFs as follows:

$$
\begin{aligned}
\mathrm{F}_{1}(\mathrm{x}) & =0, \quad \mathrm{x}<\mathrm{a} \\
& =(\mathrm{x}-\mathrm{a}) /(\mathrm{b}-\mathrm{a}), \quad \mathrm{a} \leq \mathrm{x}<\mathrm{b} \\
& =1, \quad \mathrm{~b} \leq \mathrm{x} \\
\mathrm{F}_{2}(\mathrm{x}) & =0, \quad \mathrm{x}<\mathrm{b} \\
& =(\mathrm{x}-\mathrm{b}) /(\mathrm{c}-\mathrm{b}), \quad \mathrm{b} \leq \mathrm{x}<\mathrm{c} \\
& =1, \quad \mathrm{c} \leq \mathrm{x}
\end{aligned}
$$

Recall that $F_{1}$ and $F_{2}$ are equal to various probabilities as described for Case 1 and Case 2. We now look at couple of different examples.

Example 1. Let us assume that the triplet $[a, b, c]$ in Figure 2 is $[1,2,3]$. This corresponds to a symmetric TFN. Table 1 shows specific values for $\mathrm{x}$ and the corresponding fuzzy "presumption" levels for $\Pi(\mathrm{x})$. The gray areas show $\Pi(\mathrm{x})$ and ranges for $\mathrm{P}\left(\mathrm{A}_{2}\right)$ and $\mathrm{P}\left(\mathrm{A}_{1}\right)$, calculated according to equations (18) and (21). For Case 1 , the probability $\mathrm{P}\left(\mathrm{A}_{1}\right)$ is fixed and unique for a fixed $\mathrm{x}$. On the other hand, the probability $\mathrm{P}\left(\mathrm{A}_{2}\right)$ is not unique and $\mathrm{P}\left(\mathrm{A}_{2}\right)$ resides in a range given in (18). Table 1 indicates that as well. We observe that for small values of $\Pi(\mathrm{x})$ (low level of fuzzy "presumption") the corresponding range of $\mathrm{P}\left(\mathrm{A}_{2}\right)$ is wider (more uncertainty), and for bigger values of $\Pi(\mathrm{x})$ (high fuzzy "presumption" level), range of $\mathrm{P}\left(\mathrm{A}_{2}\right)$ is narrower (less uncertainty). This makes perfect intuitive sense as well. We have the same situation for Case 2, except that the non unique probability is now $\mathrm{P}\left(\mathrm{A}_{1}\right)$.

Table 1. Example 1

\begin{tabular}{|c|c|c|c|c|c|c|c|c|c|}
\hline $\mathbf{x}$ & $\mathbf{F}_{\mathbf{1}}$ & $\mathbf{F}_{\mathbf{2}}$ & $\boldsymbol{\Pi}$ & $\mathbf{P}_{\mathbf{1 M}}$ & $\mathbf{P}_{\mathbf{1 m}}$ & $\Delta \mathbf{P}_{\mathbf{1}}$ & $\mathbf{P}_{\mathbf{2 m}}$ & $\mathbf{P}_{\mathbf{2 M}}$ & $\Delta \mathbf{P}_{\mathbf{2}}$ \\
\hline 0.5 & 0 & 0 & 0 & 0 & 0 & 0 & 0 & 1 & 1 \\
\hline 1 & 0 & 0 & 0 & 0 & 0 & 0 & 0 & 1 & 1 \\
\hline 1.2 & 0.2 & 0 & 0.2 & 0.2 & 0.2 & 0 & 0 & 0.8 & 0.8 \\
\hline 1.4 & 0.4 & 0 & 0.4 & 0.4 & 0.4 & 0 & 0 & 0.6 & 0.6 \\
\hline 1.6 & 0.6 & 0 & 0.6 & 0.6 & 0.6 & 0 & 0 & 0.4 & 0.4 \\
\hline 1.8 & 0.8 & 0 & 0.8 & 0.8 & 0.8 & 0 & 0 & 0.2 & 0.2 \\
\hline 2 & 1 & 0 & 1 & 1 & 1 & 0 & 0 & 0 & 0 \\
\hline 2.1 & 1 & 0.1 & 0.9 & 1 & 0.9 & 0.1 & 0.1 & 0.1 & 0 \\
\hline 2.2 & 1 & 0.2 & 0.8 & 1 & 0.8 & 0.2 & 0.2 & 0.2 & 0 \\
\hline 2.3 & 1 & 0.3 & 0.7 & 1 & 0.7 & 0.3 & 0.3 & 0.3 & 0 \\
\hline 2.5 & 1 & 0.5 & 0.5 & 1 & 0.5 & 0.5 & 0.5 & 0.5 & 0 \\
\hline 2.7 & 1 & 0.7 & 0.3 & 1 & 0.3 & 0.7 & 0.7 & 0.7 & 0 \\
\hline 3 & 1 & 1 & 0 & 1 & 0 & 1 & 1 & 1 & 0 \\
\hline 3.5 & 1 & 1 & 0 & 1 & 0 & 1 & 1 & 1 & 0 \\
\hline
\end{tabular}

Example 2. We now change the triplet $[\mathrm{a}, \mathrm{b}, \mathrm{c}]$ in Figure 2 to $[5,10,20]$. This corresponds to a non symmetric TFN with a larger spread of $x$. Table 2 has the corresponding numerical values. The same comments apply as in Example 1. Note that the values of $\Pi(\mathrm{x})$ and probability ranges $\Delta \mathrm{P}(\mathrm{A})$ are equivalent to Example 1 (confirming $\mathrm{x}$ and fuzzy presumption invariance).

Example 3. For Example 3 we choose a fuzzy distribution described by a half circle with the triplet $[a, b, c]=[1,2,3]$ where " $b$ " is at the circle center on $x$ axis, with the radius 1 , and outside of $[1,2,3]$ distribution is 0 . For $1 \leq \mathrm{x}$ $<3$ :

$$
\Pi(x)=\sqrt{ }\left[1-(x-2)^{2}\right]
$$

Table 3 has the results. Note that the values of $\Pi(x)$ are not linearly (uniformly) distributed, as is the case in Examples 1 and 2 . The distribution changes the fastest immediately right from $\mathrm{x}=1$ and left from $\mathrm{x}=3$, as in Table 3 .

Example 4. Final example deals with a fuzzy distribution similar to the last diagram in Figure 8. We will implement it by two quarter circles, of radius 1 , centered at $(1,1)$ and $(3,1)$ as follows:

$$
\begin{array}{cc}
\Pi(\mathrm{x})=1-\sqrt{ }\left[1-(\mathrm{x}-1)^{2}\right], & 1 \leq \mathrm{x}<2 \\
=1-\sqrt{ }\left[1-(\mathrm{x}-3)^{2}\right], & 2 \leq \mathrm{x}<3
\end{array}
$$


Table 2. Example 2

\begin{tabular}{|c|c|c|c|c|c|c|c|c|c|}
\hline $\mathbf{x}$ & $\mathbf{F}_{\mathbf{1}}$ & $\mathbf{F}_{\mathbf{2}}$ & $\boldsymbol{\Pi}$ & $\mathbf{P}_{\mathbf{1 M}}$ & $\mathbf{P}_{\mathbf{1 m}}$ & $\Delta \mathbf{P}_{\mathbf{1}}$ & $\mathbf{P}_{\mathbf{2 m}}$ & $\mathbf{P}_{\mathbf{2 M}}$ & $\Delta \mathbf{P}_{\mathbf{2}}$ \\
\hline 4 & 0 & 0 & 0 & 0 & 0 & 0 & 0 & 1 & 1 \\
\hline 5 & 0 & 0 & 0 & 0 & 0 & 0 & 0 & 1 & 1 \\
\hline 6 & 0.2 & 0 & 0.2 & 0.2 & 0.2 & 0 & 0 & 0.8 & 0.8 \\
\hline 7 & 0.4 & 0 & 0.4 & 0.4 & 0.4 & 0 & 0 & 0.6 & 0.6 \\
\hline 8 & 0.6 & 0 & 0.6 & 0.6 & 0.6 & 0 & 0 & 0.4 & 0.4 \\
\hline 9 & 0.8 & 0 & 0.8 & 0.8 & 0.8 & 0 & 0 & 0.2 & 0.2 \\
\hline 10 & 1 & 0 & 1 & 1 & 1 & 0 & 0 & 0 & 0 \\
\hline 12 & 1 & 0.2 & 0.8 & 1 & 0.8 & 0.2 & 0.2 & 0.2 & 0 \\
\hline 14 & 1 & 0.4 & 0.6 & 1 & 0.6 & 0.4 & 0.4 & 0.4 & 0 \\
\hline 16 & 1 & 0.6 & 0.4 & 1 & 0.4 & 0.6 & 0.6 & 0.6 & 0 \\
\hline 18 & 1 & 0.8 & 0.2 & 1 & 0.2 & 0.8 & 0.8 & 0.8 & 0 \\
\hline 20 & 1 & 1 & 0 & 1 & 0 & 1 & 1 & 1 & 0 \\
\hline 25 & 1 & 1 & 0 & 1 & 0 & 1 & 1 & 1 & 0 \\
\hline
\end{tabular}

Table 3. Example 3

\begin{tabular}{|c|c|c|c|c|c|c|c|c|c|}
\hline $\mathbf{X}$ & $\mathbf{F}_{\mathbf{1}}$ & $\mathbf{F}_{\mathbf{2}}$ & $\boldsymbol{\Pi}$ & $\mathbf{P}_{\mathbf{1 M}}$ & $\mathbf{P}_{\mathbf{1 m}}$ & $\Delta \mathbf{P}_{\mathbf{1}}$ & $\mathbf{P}_{\mathbf{2 m}}$ & $\mathbf{P}_{\mathbf{2 M}}$ & $\Delta \mathbf{P}_{\mathbf{2}}$ \\
\hline .5 & 0 & 0 & 0 & 0 & 0 & 0 & 0 & 1 & 1 \\
\hline 1 & 0 & 0 & 0 & 0 & 0 & 0 & 0 & 1 & 1 \\
\hline 1.2 & 0.6 & 0 & 0.6 & 0.6 & 0.6 & 0 & 0 & 0.4 & 0.4 \\
\hline 1.4 & 0.8 & 0 & 0.8 & 0.8 & 0.8 & 0 & 0 & 0.2 & 0.2 \\
\hline 1.6 & 0.9 & 0 & 0.9 & 0.9 & 0.9 & 0 & 0 & 0.1 & 0.1 \\
\hline 1.8 & .98 & 0 & .98 & .98 & .98 & 0 & 0 & 0.02 & 0.02 \\
\hline 2 & 1 & 0 & 1 & 1 & 1 & 0 & 0 & 0 & 0 \\
\hline 2.2 & 1 & 0.6 & .98 & 1 & 0.98 & 0.02 & 0.6 & 0.6 & 0 \\
\hline 2.4 & 1 & 0.8 & 0.9 & 1 & 0.9 & 0.1 & 0.8 & 0.8 & 0 \\
\hline 2.6 & 1 & 0.9 & 0.8 & 1 & 0.8 & 0.2 & 0.9 & 0.9 & 0 \\
\hline 2.8 & 1 & .98 & 0.6 & 1 & 0.4 & 0.4 & .98 & .98 & 0 \\
\hline 3 & 1 & 1 & 0 & 1 & 0 & 1 & 1 & 1 & 0 \\
\hline 3.5 & 1 & 1 & 0 & 1 & 0 & 1 & 1 & 1 & 0 \\
\hline
\end{tabular}

Table 4. Example 4

\begin{tabular}{|c|c|c|c|c|c|c|c|c|c|}
\hline $\mathbf{X}$ & $\mathbf{F}_{\mathbf{1}}$ & $\mathbf{F}_{\mathbf{2}}$ & $\boldsymbol{\Pi}$ & $\mathbf{P}_{\mathbf{1 M}}$ & $\mathbf{P}_{\mathbf{1 m}}$ & $\Delta \mathbf{P}_{\mathbf{1}}$ & $\mathbf{P}_{\mathbf{2 m}}$ & $\mathbf{P}_{\mathbf{2 M}}$ & $\Delta \mathbf{P}_{\mathbf{2}}$ \\
\hline .5 & 0 & 0 & 0 & 0 & 0 & 0 & 0 & 1 & 1 \\
\hline 1 & 0 & 0 & 0 & 0 & 0 & 0 & 0 & 1 & 1 \\
\hline 1.2 & .02 & 0 & .02 & .02 & .02 & 0 & 0 & .98 & .98 \\
\hline 1.4 & .08 & 0 & .08 & .08 & .08 & 0 & 0 & .92 & .92 \\
\hline 1.6 & .2 & 0 & .2 & .2 & .2 & 0 & 0 & .8 & .8 \\
\hline 1.8 & .4 & 0 & .4 & .4 & .4 & 0 & 0 & .6 & .6 \\
\hline 2 & 1 & 0 & 1 & 1 & 1 & 0 & 0 & 0 & 0 \\
\hline 2.2 & 1 & .02 & .98 & 1 & .98 & .02 & .02 & .02 & 0 \\
\hline 2.4 & 1 & .08 & .92 & 1 & .92 & .08 & .08 & .08 & 0 \\
\hline 2.6 & 1 & .2 & .8 & 1 & .8 & .2 & .2 & .2 & 0 \\
\hline 2.8 & 1 & .4 & .6 & 1 & .6 & .4 & .4 & .4 & 0 \\
\hline 3 & 1 & 1 & 0 & 1 & 0 & 1 & 1 & 1 & 0 \\
\hline 3.5 & 1 & 1 & 0 & 1 & 0 & 1 & 1 & 1 & 0 \\
\hline
\end{tabular}

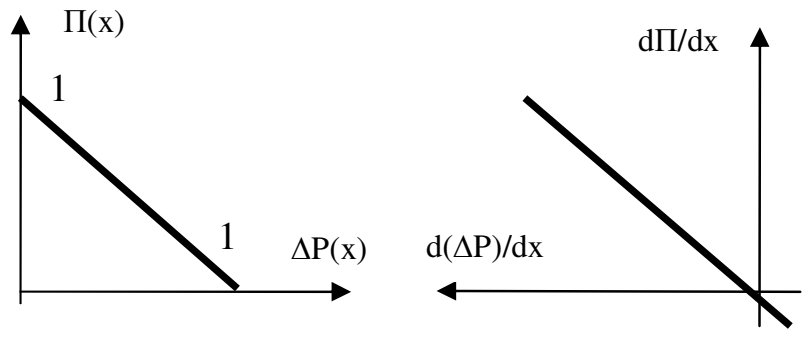

Figures 9: $\Pi(\mathrm{x})$ vs $\Delta \mathrm{P}(\mathrm{x})$, and $\mathrm{d} \Pi / \mathrm{dx}$ vs $\mathrm{d}(\Delta \mathrm{P}) / \mathrm{dx}$

Note that the values of $\Pi(x)$ in Example 4 are not linearly distributed, as expected, and that the distribution changes faster for values near $\mathrm{x}=2$, on both sides. Table 4 confirms that observation.

Based on the Examples presented, and the key results in Section5, we have the two diagrams in Figure 9. The first diagram shows how $\Pi(\mathrm{x})$ changes with $\Delta \mathrm{P}$, for any fuzzy distribution. This is simple consequence of Theorem 2 and presumption and $\mathrm{x}$-invariant nature of it. The second diagram in Figure 9 indicates the relationship between $\mathrm{d} \Pi / \mathrm{dx}$ and $\mathrm{d}(\Delta \mathrm{P}) / \mathrm{dx}$, based on Corollary 2. The diagrams are universal for any distribution $\Pi(\mathrm{x})$.

Theorem 2 and Corollary 2 are confirmed numerically in all the examples. The Equations (36)-(38) or any other form of $\Pi(x)$, with their derivatives for the density functions, if they exist, indicate how to practically generate fuzzy distributions out of corresponding non unique random distributions, as well as how to do uncertainty alignment for a specific case at hand.

\section{CONCLUSION}

In this paper we define new fuzzy to random uncertainty alignment methodology, in which fuzziness can be described as non unique randomness. We employ the most basic properties of random and fuzzy distributions for this result and give precise both upper and lower bounds of changes in random distributions, required to produce data fuzziness. The range of randomness of the corresponding probabilistic events is a function of fuzzy distribution presumption level and it holds fo any fuzzy distribution. The main results can be considered as an universal fuzzyrandom (possibilistic-probabilistic) uncertainty alignment law which is fuzzy presumption-invariant and fuzzy argument $\mathrm{x}$ invariant for any given distribution. The result can be employed effectively in a variety of data fusion and decision problems where both objective (hard, random, 
probabilistic, sensor based) data are to be fused with subjective (soft, fuzzy, possibilistic, human based) data [9], [10]. One way to interpret the results is as a precise mathematical description of fuzzy-to-random "consistency principle" first introduced by Zadeh in his classic paper [2], as a loose and intuitive notion. In a future work we will extend the results in specific areas such as machinehuman data fusion. Also, further properties of both random and fuzzy data will be analyzed in the light of the paper's main results. In particular we will consider relationship between probabilistic and possibilistic axioms in the light of this paper results.

\section{REFERENCES}

[1] A. Leon-Garcia, Probability, Statistics, and Random Processes for Electrical Engineering, $3^{\text {rd }}$ Edition, Pearson Education Inc., 2008.

[2] L. A. Zadeh, Fuzzy sets as a basis for a theory of possibility, Fuzzy Sets and Systems 1, 3-28, 1978.

[3] D. Dubois and H. Prade, Possibility theory, Probability theory and multiple valued logics: A clarification, Annals of Mathematics and Artificial Intelligence, Vol. 32, 35-66, 2002.

[4] A. Kaufmann and M. M. Gupta, Introduction to Fuzzy Arithmetic, Theory and Applications, Van Nostrand Reinhold, 1985.

[5] H. J. Zimmermann, Fuzzy Set Theory and its Applications, $4^{\text {th }}$ Edition, Kluwer Academic Publishers, 2001.

[6] F. P.A. Coolen et all, Imprecise probability, Int'l Encyclopedia of Statistical Science, Springer, 2010.

[7] Ruud van der Helm, Towards a clarification of probability, possibility and plausibility: how semantics could help futures practice to improve, Foresight, Vol. 8, No.3, 17-27, 2008.

[8] P. Agarwal and H.S. Najal, Possibility theory vs possibility theory in fuzzy measure theory, P. Agarwal Int. Journal of Engineering Research and Applications, Vol.5, No.5, 37-43, 2015.

[9] R. Bellman and L. Zadeh, Decision-Making in a fuzzy environment, Management Science, Vol. 17, No. 4, 1979.

[10] M. P. Jenkins et all, Towards context aware data fusion: Modeling and integration of situationally qualified human observations to manage uncertainty in a hard-soft fusion process, Information Fusion, Vol. 21, 130-144, 2015. 